\title{
Preserving preference rankings under non-financial background
}

$$
\text { risk }^{*}
$$

\author{
Y. Malevergne $\mathrm{e}^{a, b, c}$ and B. Rey ${ }^{d}$ \\ ${ }^{a}$ University of Saint-Etienne - ISEAG, France \\ ${ }^{b}$ EMLYON Business School - Cefra, France \\ ${ }^{c}$ ETH Zurich, Switzerland \\ ${ }^{d}$ University of Lyon, University Lyon 1 - ISFA, France \\ e-mails: ymalevergne@ethz.ch and rey-fournier@univ-lyon1.fr
}

JEL classification: D81

Keywords: Decision analysis, Multivariate risk, Background risk, Disaggregation of harms, Risk independence

${ }^{*}$ The authors acknowledge helpful discussions and exchanges with L. Eeckhoudt, H. Schlesinger and B. Versaevel. All remaining errors are ours. 


\title{
Preserving preference rankings under non-financial background risk
}

\begin{abstract}
We investigate the impact of a non-financial background risk $\tilde{\varepsilon}$ on the preference rankings between two independent financial risks $\tilde{z}_{1}$ and $\tilde{z}_{2}$ for an expected-utility maximizer. More precisely, we provide necessary and sufficient conditions for the alternative $\left(x_{0}+\tilde{z}_{1}, y_{0}+\tilde{\varepsilon}\right)$ to be preferred to $\left(x_{0}+\tilde{z}_{2}, y_{0}+\tilde{\varepsilon}\right)$ whenever $\left(x_{0}+\tilde{z}_{1}, y_{0}\right)$ is preferred to $\left(x_{0}+\tilde{z}_{2}, y_{0}\right)$. Utility functions that preserve the preference rankings are fully characterized. Their practical relevance is discussed in light of recent results on the constraints for the modeling of the preference for the disaggregation of harms.
\end{abstract}

JEL classification: D81

Keywords: Multivariate risk, Background risk, Disaggregation of harms, Risk independence 


\section{Introduction}

Decision making under uncertainty often takes place in the context of multiple risks. Consequently, decisions about endogenous risks must be made while simultaneously facing one (or more) exogenous background risk(s). However, it is often assumed, both in the academic literature and in practice, that only the main risk factor of a specific problem must be accounted for. Such a simplifying approach is quite common and mainly relies on the belief that the effects of additional sources of risks are of second order with respect to the consequences of the main risk factor. Along this line, many standard decision models have been developed under the assumption of the existence of one single source of risk. It is therefore important to investigate the extent to which the models developed under this single-risk assumption are robust in the presence of multiple risks.

In an economic context, Gollier and Schlesinger (2003) have recently started addressing this issue in the particular case of a purely (additive) financial risk, i.e, when the background risk is of the same kind as the main risk. The objective of this note is to extend their investigation to the context of multivariate risks in order to explicitly account for the existence of risks of different natures. Among the many applications in which the background risk is not of the same nature as the endogenous decision variables, we can, for example, refer to one-period models in which the background risk is a non-financial risk say, an environmental risk, or a health risk (on this, see Bleichrodt et al. (2003) for recent results). But the exogenous risk can also affect a non-consumable pension-asset, or the supply of labor, or an economic state variable, or the future consumption (see the books by Gollier (2001) and Eeckhoudt et al. (2005)). Applications can also refer the more recent problem, investigated by Franke et al. (2006), of a multiplicative background risk in order to account, among others, for the random behavior of interest rates. In a broader context, Leskinen and Kangas (2005) have recently investigated the question of rank reversals in multi-criteria decision problems when the performance of decision alternatives is analyzed by use of ratio-scale pairwise comparisons.

Formally, the question we address is the following:

"When can one say that an expected-utility maximizer prefers the alternative $\left(x_{0}+\right.$ $\left.\tilde{z}_{1}, y_{0}+\tilde{\varepsilon}\right)$ to the alternative $\left(x_{0}+\tilde{z}_{2}, y_{0}+\tilde{\varepsilon}\right)$ whenever she prefers $\left(x_{0}+\tilde{z}_{1}, y_{0}\right)$ to $\left(x_{0}+\right.$

$\left.\tilde{z}_{2}, y_{0}\right)$, provided that the non-financial background risk $\tilde{\varepsilon}$ is independent of $\tilde{z}_{1}$ and $\tilde{z}_{2}$."

In addition to its theoretical relevance, this problem addresses the practical issue of the direction in which preferences are affected by the presence of a new independent source of unavoidable risk. Consider for instance a firm that faces two investment opportunities whose future cash-flows are respectively $\tilde{z}_{1}$ and $\tilde{z}_{2}$ with the alternative $\tilde{z}_{1}$ preferred to the alternative $\tilde{z}_{2}$ in the absence of any secondary source of risk. Answering our question tells us explicitly how the decision of the firm will be affected by the presence 
of the background risk $\tilde{\varepsilon}$, which can be seen as any kind of risk the firm has to face up: the future evolution of the environmental legislation (e.g., carbon dioxide constraints, Busch and Volker (2007)), the financial disclosures and their certification (Sarbanes-Oxley Act, Chhaochharia and Grinstein (2007)), interest rates and financing conditions (Franke et al. (2006)), changes in health and sanitary regulations (asbestos or cigarettes, Mullin and Malani (2004)), and so on. Individuals obviously face similar multirisks decision problems. This issue is therefore particularly relevant both in theory and practice.

As we shall see, the family of utility functions that are solutions to this problem does not boil down to the set of utility functions that only depend on the marginal distributions of their attributes (and, therefore, not on their joint probability distribution), namely the set of additive utility functions (Fishburn (1967)). Indeed, it appears that the class of utility functions that solves the problem does not simply restrict to the utility functions for which the non-pecuniary variable does not impact the marginal utility of wealth (cross derivative equal to zero). More precisely, we show that the class of the so-called risk-independent utility functions, introduced in the early seventies by Keeney $(1971,1973)$ in relation to the concepts of conditional risk aversion and conditional risk premium, constitutes the solution to this problem ${ }^{1}$. However, by restricting to an actuarially neutral background risk, in order to disentangle the pure risk effect from the level effect, we find that the set of admissible utility functions is broader.

The note is organized as follows. Section 2 answers the main question of the preservation of the preference rankings in the presence of a non-financial risk, firstly in the general case, i.e., when one does not impose any restriction on the mean value of the background risk, and secondly, when one focuses on an actuarially neutral background risk in order to disentangle the level effect and the pure risk effect. We show that the class of utility functions derived in the absence of any constraint on the background risk is then enlarged by a family of functions that constitutes a non-trivial generalization of the one-dimensional quadratic functions obtained by Gollier and Schlesinger (2003) for an additive financial background risk. Section 3 provides several examples of applications of our results to special cases: $(l)$ we derive the result obtained by Gollier and Schlesinger (2003) for an additive financial background risk as a special case of our result, ( $(l)$ we extend on the results in Franke et al. (2006) to show that the family of utility functions that preserve preference rankings under a multiplicative background risk is the constant relative risk aversion family and ( $u l l)$ we show that there is no (risk-averse) utility function that preserves preference rankings in the presence of both an additive and a multiplicative background risk. Then, as a corollary to the main results of this note, section 4 shows that the class of utility functions that preserve preference rankings is compatible with the modeling of the preferences of an individual for the disaggregation, or the aggregation, of harms, according to the assumptions made on the sign of the cross derivative of the utility function (Eeckhoudt et al. (2007)). It proves that this class of utility functions possesses the

\footnotetext{
${ }^{1}$ See also Keeney and Raiffa (1976) which consider related utility independence conditions.
} 
minimum properties one can expect. It also means that this class of utility functions can be very useful when one faces a multidimensional decision problem for which it is known that the preference rankings on one of the variable are not impacted by the presence of the other variable(s). Section 5 offers a brief conclusion.

\section{Conditions on utility to preserve preference rankings}

We consider an individual with a two-arguments von Neumann-Morgenstern utility function $U$ (assumed strictly increasing with respect to each argument, concave and $n$-times continuously differentiable). We will assume, for illustration purpose and for ease of the exposition, that the first argument refers to the agent's wealth while the second one refers to any background risk (of a financial or a non-financial nature, as exemplified in introduction). Thus, we will denote by $\mathrm{E}\left[U\left(x_{0}+\tilde{z}, y_{0}+\tilde{\varepsilon}\right)\right]$ the individual's expected utility where $\left(x_{0}, y_{0}\right)$ represents her initial endowment, $\tilde{z}$ is a random wealth payoff and $\tilde{\varepsilon}$ a background risk. Notice immediately that our restriction to the bivariate case, namely the case for which there is one main risk factor and only one background risk is just for notational convenience. Our results generalize naturally to the situation where the decision maker faces several background risks. In such a case, the initial non-pecuniary endowment reads $y_{0}=\left(y_{0,1}, \ldots, y_{0, p}\right)$ while the background risk becomes $\tilde{\varepsilon}=\left(\tilde{\varepsilon}_{1}, \ldots, \tilde{\varepsilon}_{p}\right)$.

Throughout this note, we assume that the two risks $\tilde{z}$ and $\tilde{\varepsilon}$ are independent. We could be blamed for such a simplifying assumption since any interaction between these two risks would play a role on the preference rankings. But in this case, the impact of the second risk on the first one would be blatant. Besides, in the absence of any restriction on the dependence between the two risks, the set of utility functions preserving preference rankings clearly restricts to the set of additive utility functions ${ }^{2}$. That is why we focus, in the sequel, on the case where both risks are independent of one another, so that the potential changes induced by the presence of the exogenous risk does not come from a direct interaction with the endogenous one but specifically from the bivariate nature of the decision problem and the properties of the utility function of the economic agent.

Considering two random wealth payoffs $\tilde{z}_{1}$ and $\tilde{z}_{2}$ and an independent random variable $\tilde{\varepsilon}$ representing the background risk acting on the non-pecuniary dimension of the agent's welfare, our main result is

Proposition 1. The two following statements are equivalent:

$$
\text { (ı) }\left(x_{0}+\tilde{z}_{1}, y_{0}\right) \succeq\left(x_{0}+\tilde{z}_{2}, y_{0}\right) \Longrightarrow\left(x_{0}+\tilde{z}_{1}, y_{0}+\tilde{\varepsilon}\right) \succeq\left(x_{0}+\tilde{z}_{2}, y_{0}+\tilde{\varepsilon}\right), \forall \tilde{z}_{i} \text { and } \forall \tilde{\varepsilon} \text { independent }
$$

\footnotetext{
${ }^{2}$ The fact that any additive utility function $U(x, y)=u_{x}(x)+u_{y}(y)$ preserves preference rankings on the first argument in the presence of any background risk on the second argument, irrespective of their dependence, is obvious. The necessity almost straightforwardly results from the considerations of Pollak (1967) on the so-called k-standard lottery tickets for which the preservation of preference rankings must hold.
} 
of $\tilde{z}_{i}$,

(ul) the bivariate utility function reads $U(x, y)=F(x) \cdot G(y)+h(y)$, where $G(\cdot)$ is any function with a constant sign.

Before we prove this assertion, let us remark that, following the definition introduced by Keeney $(1971,1973)$, the utility functions satisfying statement $(l l)$ are such that the financial risk is risk-independent of the non-financial risk. It is also equivalent to say that the utility function $U(x, y)$ in $(u l)$ satisfies

$$
\frac{\partial}{\partial y}\left(-\frac{U_{11}(x, y)}{U_{1}(x, y)}\right)=0, \quad \forall(x, y)
$$

Keeney (1971, theorem 1) has established that utility functions that satisfy (1) can be completely specified by the knowledge of three conditional utility functions $U\left(x_{0}, y\right), U\left(x_{1}, y\right)$ and $U\left(x, y_{0}\right)$ for arbitrary $x_{0}, x_{1} \neq x_{0}$ and $y_{0}$. Defining $x_{0}$ and $x_{1}$ such that $U\left(x_{1}, y_{0}\right)>U\left(x_{0}, y_{0}\right)$ and then arbitrarily setting the origin and units of measure of $U(x, y)$ by $U\left(x_{0}, y_{0}\right)=0$ and $U\left(x_{1}, y_{0}\right)=1$, we get

$$
U(x, y)=U\left(x_{0}, y\right)\left[1-U\left(x, y_{0}\right)\right]+U\left(x_{1}, y\right) \cdot U\left(x, y_{0}\right)
$$

Proof of proposition 1. The sufficiency of the risk-independence property for preserving preference rankings is obvious. Indeed, from $(u)$, we can assert that $\left(x_{0}+\tilde{z}_{1}, y_{0}\right) \succeq\left(x_{0}+\tilde{z}_{2}, y_{0}\right)$ implies that $\mathrm{E}\left[F\left(x_{0}+\tilde{z}_{1}\right)\right] \cdot G\left(y_{0}\right) \geq \mathrm{E}\left[F\left(x_{0}+\tilde{z}_{2}\right)\right] \cdot G\left(y_{0}\right)$. Then, multiplying both sides of this last equation by $\mathrm{E}\left[G\left(y_{0}+\tilde{\varepsilon}\right)\right] / G\left(y_{0}\right)$, which is positive by assumption, and accounting for the independence of $\tilde{z}_{i}$ and $\tilde{\varepsilon}$, the statement that $\left(x_{0}+\tilde{z}_{1}, y_{0}\right) \succeq\left(x_{0}+\tilde{z}_{2}, y_{0}\right)$ implies $\mathrm{E}\left[U\left(x_{0}+\tilde{z}_{1}, y_{0}+\tilde{\varepsilon}\right)\right] \geq \mathrm{E}\left[U\left(x_{0}+\tilde{z}_{2}, y_{0}+\tilde{\varepsilon}\right)\right]$, which is the same as $\left(x_{0}+\tilde{z}_{1}, y_{0}+\tilde{\varepsilon}\right) \succeq\left(x_{0}+\tilde{z}_{2}, y_{0}+\tilde{\varepsilon}\right)$. It concludes the proof of the sufficiency.

As for the proof of the necessity, let us remark that in order for statement $(\imath)$ to hold for any risk $\tilde{\varepsilon}$, it must at least hold for a degenerate $\tilde{\varepsilon}$, i.e., for a constant $\tilde{\varepsilon}=\varepsilon$. Therefore, the alternative $\left(x_{0}+\tilde{z}_{1}, y_{0}+\varepsilon\right)$ must always be preferred to the alternative $\left(x_{0}+\tilde{z}_{2}, y_{0}+\varepsilon\right)$ irrespective of the fixed, but arbitrary, value of $\varepsilon$. In other words, it is necessary that the decision maker's preferences over any financial lotteries for a fixed value of the non-financial argument are the same regardless of the amount of the non-financial argument. Consequently, the risk-independence property (1) - and equivalently $(l l)$ - is necessary for $(l)$ to hold. Besides, since the utility function $U$ is such that $U_{1}(x, y)=F^{\prime}(x) \cdot G(y)$ is positive for all $x$ and $y$, the function $G$ must keep a constant sign.

Accounting for any form of background risk $\tilde{\varepsilon}$ has a severe drawback in so far as it mixes two effects: a level-effect (analogous to the wealth-effect for a financial risk) related to the amplitude of the mean value of $\tilde{\varepsilon}$ and a pure-risk effect related to the shape of the background risk distribution (more or less skewed, more or less fat-tailed, and so on), irrespective of its mean value. It is thus desirable to focus on 
actuarially neutral background risks in order to have a better understanding of the impact of each of these effects. Under such a restriction on the distribution of the background risk, the result hereafter holds (see the Appendix for the proof).

Proposition 2. The two following statements are equivalent:

(ı) $\left(x_{0}+\tilde{z}_{1}, y_{0}\right) \succeq\left(x_{0}+\tilde{z}_{2}, y_{0}\right) \Longrightarrow\left(x_{0}+\tilde{z}_{1}, y_{0}+\tilde{\varepsilon}\right) \succeq\left(x_{0}+\tilde{z}_{2}, y_{0}+\tilde{\varepsilon}\right), \forall \tilde{z}_{i}$ and $\forall \tilde{\varepsilon}$ independent of $\tilde{z}_{i}$ with $\mathrm{E}[\tilde{\varepsilon}]=0$,

(ul) the bivariate utility function reads $U(x, y)=F(x) \cdot G(y)+h(y)$, where G keeps a constant sign, or $U(x, y)=F(x) \cdot y+h(y)+g(x)$.

To compare, in the case of a one-argument utility function, when restricted to an actuarially neutral background risk, Gollier and Schlesinger (2003) show that, in addition to CARA utility functions, the quadratic utility functions are admissible. Note that in the present (bi-dimensional) case, the functions of the form $U(x, y)=F(x) \cdot y+h(y)+g(x)$ constitute a non trivial generalization of their result.

We remark that the two sets of utility functions that preserve preference rankings have different implications in terms of decision analysis. Indeed, utility functions $U(x, y)=F(x) \cdot y+h(y)+g(x)$ are such that if, for some level $x_{0}$ of the first attribute, the outcome $y_{0}+\tilde{\varepsilon}_{1}$ is preferred to the outcome $y_{0}+\tilde{\varepsilon}_{2}$, where $\tilde{\varepsilon}_{1}$ and $\tilde{\varepsilon}_{2}$ are independent actuarially neutral background risks then the outcome $y_{0}+\tilde{\varepsilon}_{1}$ will be preferred to $y_{0}+\tilde{\varepsilon}_{2}$ for any level $x$ of the first attribute,

$$
\left(x_{0}, y_{0}+\tilde{\varepsilon}_{1}\right) \succeq\left(x_{0}, y_{0}+\tilde{\varepsilon}_{2}\right) \Longrightarrow\left(x, y_{0}+\tilde{\varepsilon}_{1}\right) \succeq\left(x, y_{0}+\tilde{\varepsilon}_{2}\right), \forall x
$$

The proof simply amounts to notice that $\mathrm{E}\left[U\left(x_{0}, y_{0}+\tilde{\varepsilon}_{1}\right)\right]-\mathrm{E}\left[U\left(x_{0}, y_{0}+\tilde{\varepsilon}_{2}\right)\right]=\mathrm{E}\left[h\left(y_{0}+\tilde{\varepsilon}_{1}\right)\right]-$ $\mathrm{E}\left[h\left(y_{0}+\tilde{\varepsilon}_{2}\right)\right]$ does not depend on the value of the first attribute.

On the contrary, the set of risk-independent utility functions may exhibit an alternation in the preference rankings when the level of the first attribute varies. Indeed, in such a case $\mathrm{E}\left[U\left(x_{0}, y_{0}+\tilde{\varepsilon}_{1}\right)\right]-$ $\mathrm{E}\left[U\left(x_{0}, y_{0}+\tilde{\varepsilon}_{2}\right)\right]=F\left(x_{0}\right) \cdot\left(\mathrm{E}\left[G\left(y_{0}+\tilde{\varepsilon}_{1}\right)\right]-\mathrm{E}\left[G\left(y_{0}+\tilde{\varepsilon}_{2}\right)\right]\right)+\left(\mathrm{E}\left[h\left(y_{0}+\tilde{\varepsilon}_{1}\right)\right]-\mathrm{E}\left[h\left(y_{0}+\tilde{\varepsilon}_{2}\right)\right]\right)$, so that the outcome $y_{0}+\tilde{\varepsilon}_{1}$ is preferred (resp. not preferred) to the outcome $y_{0}+\tilde{\varepsilon}_{2}$ according as $F\left(x_{0}\right) \gtreqless-\frac{\mathrm{E}\left[h\left(y_{0}+\tilde{\varepsilon}_{1}\right)\right]-\mathrm{E}\left[h\left(y_{0}+\tilde{\varepsilon}_{2}\right)\right]}{\mathrm{E}\left[G\left(y_{0}+\tilde{\varepsilon}_{1}\right)\right]-\mathrm{E}\left[G\left(y_{0}+\tilde{\varepsilon}_{2}\right)\right]}$. Thus, depending on the decision analysis application one has in mind, one of the two sets of utility functions can be more relevant than the other, according as the preference rankings should also be preserved in terms of (3) or not.

As for risk independent utility functions, the second set of utility functions introduced in proposition 2 can be completely specified by the knowledge of three conditional utility functions.

Proposition 3. Any bivariate utility function $U(x, y)=F(x) \cdot y+h(y)+g(x)$ can be completely specified by the knowledge of three conditional utility functions $U\left(x_{0}, y\right), U\left(x, y_{0}\right)$ and $U\left(x, y_{1}\right)$. It then 

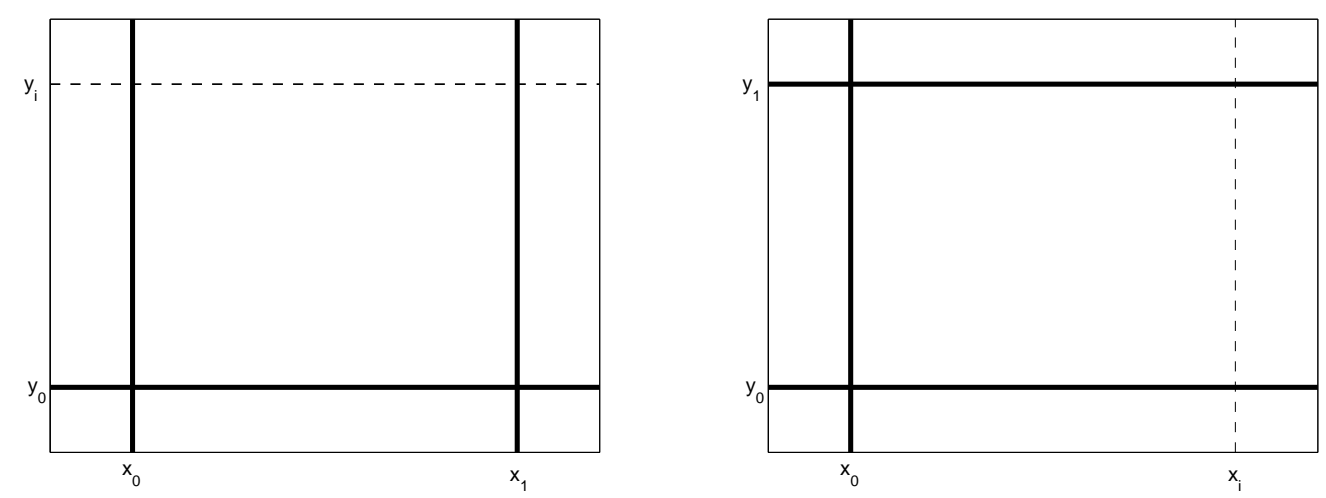

Figure 1: Schematic representation of the sets of conditional utility functions necessary to completely specify the bivariate utility function according as it is a risk-independent utility function (left panel) or the utility function considered in proposition 3 (right panel).

reads

$$
U(x, y)=U\left(x_{0}, y\right)+U\left(x, y_{0}\right)+\left[U\left(x, y_{1}\right)-U\left(x, y_{0}\right)-1\right] \cdot \frac{y-y_{0}}{y_{1}-y_{0}},
$$

for arbitrary $x_{0}, y_{0}$ and $y_{1} \neq y_{0}$ such that $U\left(x_{0}, y_{0}\right)=0$ and $U\left(x_{0}, y_{1}\right)=1$.

We prove this result in the appendix. Notice that the three conditional utility functions necessary to specify the set of utility functions in proposition 3 are not the same as the three ones that are necessary to determine the set of risk-independent utility functions (see eq. (2)). The difference is illustrated on figure 1. The relative utilities must be assessed along the heavy lines in order the fix the value of the utility along the dashed line for any $x_{i}$ or $y_{i}$, depending on the case under consideration.

\section{Applications}

We now provide three examples of applications of our results.

Example 1 (Derivation of Gollier and Schlesinger (2003)'s results). Propositions 1 and 2 constitute a natural generalization of the results obtained by Gollier and Schlesinger (2003) for a one-argument utility function. Indeed, considering the bivariate utility function $U(x, y)=u(x+y)$ one retrieves their results as a special case of propositions 1 and 2. More precisely, as shown by Gollier and Schlesinger (2003), we obtain that the preference rankings are not affected by the presence of an independent additive background risk if and only if the utility function is CARA insofar as condition (1) applied to $u(x+y)$ yields $\left[\frac{u^{\prime \prime}(z)}{u^{\prime}(z)}\right]^{\prime}=0$; hence the result. Now, if we restrict our attention to the case of an actuarially neutral background risk, the constraint $U_{122}=0$ (see Appendix) simply reads $u^{(3)}=0$ which yields the quadratic utility function. 
Example 2 (Multiplicative background risk). Extending on the results in Franke et al. (2006), it is interesting to note that in the case of a multiplicative background risk, namely when the bivariate utility function reads $U(x, y)=u(x \cdot y)$, relation (1) is satisfied if and only if $\left[\frac{z \cdot u^{\prime \prime}(z)}{u^{\prime}(z)}\right]^{\prime}=0$, so that the family of utility functions that preserve preference rankings under a multiplicative background risk is the constant relative risk aversion (CRRA) family

$$
u(z)=\frac{1}{1-\gamma} \cdot z^{1-\gamma}
$$

Since this set of utility functions is often used to model investors' choice in terms of asset allocation, we can conclude that the many results obtained under the assumption of CRRA preferences should be robust to the presence of multiplicative background risks which can easily model the inflation risk ( $y$ can be seen as the stochastic discount factor between real and nominal values), or the tax risk ( $x$ is the pre-tax income while $x \cdot y$ provides the post-tax income) and so on.

Focusing on an actuarially neutral background risk ${ }^{3}$, the constraint $U_{122}=0$ leads to the differential equation $z \cdot u^{(3)}(z)+2 u^{\prime \prime}(z)=0$, whose solution is

$$
u(z)=a \cdot \ln z+b \cdot z,
$$

up to an affine transform. It is then particularly interesting to notice that, in this later case, among the set of utility functions that preserve preference rankings in the presence of an actuarially neutral multiplicative background risk, only the risk-neutral utility function $u(z)=z$ (case $a=0$ in eq. (5) and $\gamma=0$ in eq. (4)) and the logarithmic utility function $u(z)=\ln z$ (case $b=0$ in eq. (5) and $\gamma \rightarrow 1$ in eq. (4)) are also compatible with the preservation of the preference rankings in the presence of a multiplicative background risk in the general case. Besides, let us eventually notice that in the case of the logarithmic utility, the result still holds for a dependent background risk since it is an additive utility function for multiplicative risks.

Example 3 (Additive and multiplicative background risk). As pointed out in the introduction, the results of propositions 1 and 2 generalize to the case of a multidimensional background risk. Therefore, in the realistic case where the agent is subjected to both an additive and a multiplicative background risk (e.g. a random initial wealth and a stochastic interest rate), we must conclude that preference rankings are preserved if and only if the decision maker is risk-neutral. Indeed, a straightforward generalization of proposition 1 and equation (1) to the trivariate utility function $U\left(x, y_{1}, y_{2}\right)=u\left(y_{1} \cdot x+y_{2}\right)$, shows

\footnotetext{
${ }^{3}$ Here, it would be better to write $U(x, y)=u(x \cdot(1+y))$ with $\mathrm{E}[y]=0$ since an actuarially neutral multiplicative background risk must have a mean value equal to one instead of zero (see the discussion in Franke et al. (2006)), but this shift in the expectation does not change our conclusions.
} 
that the two requirements $\left[\frac{u^{\prime \prime}(z)}{u^{\prime}(z)}\right]^{\prime}=0$ and $\left[\frac{z \cdot u^{\prime \prime}(z)}{u^{\prime}(z)}\right]^{\prime}=0$ must be fulfilled, so that, necessarily, the coefficient of absolute risk aversion $\frac{u^{\prime \prime}(z)}{u^{\prime}(z)}$ equals zero and hence the result.

\section{Further properties}

Proposition 1 has shown that the use of the class of utility functions that satisfies the property of riskindependence is relevant if and only if the decision maker knowns a priori that among the set of decision variables she has to face up, the preference rankings on one of these variables is not impacted by the presence of the others. However, for this result to be useful in practice, it remains to check that this class of utility functions is well-behaved. Consequently, as a corollary to the main result of this note, we would like to discuss the signs of the successive cross-derivatives of the set of utility functions that are solution to our problem.

To motivate the interest of the question, recall that in a recent paper, Eeckhoudt et al. (2007) give a simple and intuitive interpretation for the meaning of such derivatives. They show that the alternation of signs of the successive cross-derivatives of the utility function corresponds to a direction on preferences. Following these authors, for a correlation-averse individual ${ }^{4}\left(U_{12}<0\right)$, preferences for the disaggregation of harms corresponds to $U_{112}>0, U_{122}>0$, and $U_{1122}<0$. In the same way, for a correlation-loving individual $\left(U_{12}>0\right)$, preferences for the aggregation of harms corresponds to $U_{112}<0, U_{122}<0$, and $U_{1122}>0$. The intuition for these results for a correlation-averse individual, for example, is the following. For such an individual, a higher level of non-pecuniary endowment mitigates the detrimental effect of a reduction in wealth. Similarly, a higher wealth level helps to temper the detrimental effects of accepting a non-pecuniary risk. Such an individual prefers also to disaggregate two harmful risks.

Let us now show that the class of utility functions defined by statement $(u l)$ in proposition 1 is compatible with the alternation of signs:

Corollary 1. The set of utility functions that satisfies the preservation of preference rankings under a non-financial background risk also satisfies the requirements for the modeling of individual preferences for the aggregation or the disaggregation of harms, according as the agent is either correlation averse or not, i.e., according as $U_{12} \lessgtr 0$.

Technically, the corollary states that the set of utility functions characterized by proposition 1 are such that either $U_{12}<0$ and $U_{112}>0$ and $U_{122}>0$ and $U_{1122}<0$, or $U_{12}>0$ and $U_{112}<0$ and

\footnotetext{
${ }^{4}$ This concept was first introduced by Richard (1975), and was explored further by Epstein and Tanny (1980).
} 
$U_{122}<0$ and $U_{1122}>0$. This result comes from the fact that, under conditions of existence, we have

$$
\frac{U_{11}(x, y)}{U_{1}(x, y)}=\frac{U_{112}(x, y)}{U_{12}(x, y)}=\frac{U_{1122}(x, y)}{U_{122}(x, y)}, \quad \forall x, y
$$

So, assuming that the absolute risk aversion for wealth $\left(-\frac{U_{11}}{U_{1}}\right)$ is positive, both $\frac{U_{112}}{U_{12}}$ and $\frac{U_{1122}}{U_{122}}$ are negative, which amounts to say that $U_{12}$ and $U_{112}$ on the one hand, and $U_{122}$ and $U_{1122}$ on the other hand, have opposite signs. Now, concerning the relative sign of $U_{112}$ and $U_{122}$, it can be determined by help of the relation

$$
\frac{U_{122}}{U_{112}}=\left(-\frac{U_{11}}{U_{1}}\right)^{-1} \cdot\left(-\frac{U_{22}}{U_{2}}\right)
$$

satisfied by the utility functions that preserve preference rankings. Hence, assuming that the individual's absolute risk aversion toward financial risks and toward non-financial risks are both positive, $U_{112}$ and $U_{122}$ must have the same sign.

As for the utility functions that preserve preference rankings under an actuarially neutral background risk, the Appendix shows that the only restriction that these utility functions have to fulfill is $U_{122}=0$. Therefore, it places no constraint on the sign (and the alternation thereof) of the derivatives of $U$, so that this family of utility functions can obviously still comply with Eeckhoudt et al. (2007)'s requirements for the preferences for the disaggregation of harms but not necessarily.

\section{Conclusion}

Economic modeling often relies on a utterly simplistic idealization of the human behavior and of his/her environment in order to yield tractable results. In particular, it is often assumed that the sources of risk are well-identified so that the environment is free from any background uncertainty. This obviously does not hold in most real-world circumstances. As detailed in this note, the introduction of a new source of uncertainty is innocuous only in the very restrictive cases where the property of risk-independence holds. As a consequence, extant results that pertain to a restricted number of well-identified sources of risk cannot be assumed to extend to models with background risks (either financial or non-financial) when this property does not apply. Nonetheless, when the second risk can be plausibly thought not to have any impact on the preference rankings of the first risk, our corollary result according to which the class of risk-independent utility functions enjoys suitable properties vis-a-vis the aggregation or the disaggregation of harms should provide additional motivations for the use of this class of utility functions for practical multicriteria decision problems, as originally advocated by Keeney. 


\section{Appendix}

Proof of proposition 2. The proof mainly follows the line of the demonstration of the second proposition of Gollier and Schlesinger (2003). It starts with generalizing the notion of derived utility function introduced by Kihlstrom et al. (1981) and Nachman (1982), which allows us to define the cross derived utility function $V$ as

$$
V(x, y)=\mathrm{E}[U(x, y+\tilde{\varepsilon})], \quad \forall x, y
$$

It simply means that an individual with the preferences described in our model and facing the two independent risks behaves as an individual with the derived utility function $V$ facing the risk $\tilde{z}$ only. With this definition, statement $(\imath)$ can be rewritten as

$$
\mathrm{E}\left[U\left(x_{0}+\tilde{z}_{1}, y_{0}\right)\right] \geq \mathrm{E}\left[U\left(x_{0}+\tilde{z}_{2}, y_{0}\right)\right] \Longrightarrow \mathrm{E}\left[V\left(x_{0}+\tilde{z}_{1}, y_{0}\right)\right] \geq \mathrm{E}\left[V\left(x_{0}+\tilde{z}_{2}, y_{0}\right)\right]
$$

This relation holds irrespective of any restriction on $\tilde{z}_{1}$ and $\tilde{z}_{2}$ if and only if

$$
-\frac{U_{11}(x, y)}{U_{1}(x, y)}=-\frac{V_{11}(x, y)}{V_{1}(x, y)}, \quad \forall x, y
$$

By definition (8), the equation above is equivalent to

$$
\mathrm{E}[f(x, y, \tilde{\varepsilon})]=0, \quad \forall x, y,
$$

where we have set $f(x, y, \varepsilon)=U_{11}(x, y) \cdot U_{1}(x, y+\varepsilon)-U_{1}(x, y) \cdot U_{11}(x, y+\varepsilon)$. Now by Jensen's inequality, it is necessary for the relation

$$
\mathrm{E}[\tilde{\varepsilon}]=0 \Longrightarrow \mathrm{E}[f(x, y, \tilde{\varepsilon})]=0, \quad \forall x, y
$$

to hold that $f$ be a linear function in $\varepsilon$, so that we must have $\partial_{\varepsilon}^{2} f=0$, which is equivalent to:

$$
U_{11}(x, y) \cdot U_{122}(x, y+\varepsilon)-U_{1}(x, y) \cdot U_{1122}(x, y+\varepsilon)=0, \quad \forall x, y \text { and } \varepsilon .
$$

Under the assumption that $U_{122} \neq 0$, this relation leads to

$$
\frac{U_{11}(x, y)}{U_{1}(x, y)}=\frac{U_{1122}(x, y+\varepsilon)}{U_{122}(x, y+\varepsilon)}, \quad \forall x, y \text { and } \varepsilon,
$$

which means that both $\frac{U_{11}(x, y)}{U_{1}(x, y)}$ and $\frac{U_{1122}(x, y)}{U_{122}(x, y)}$ are equal to the same function, say $k$, which depends of $x$ only. Obviously, any solution of $\frac{U_{11}(x, y)}{U_{1}(x, y)}=k(x)$ is also solution of $\frac{U_{1122}(x, y)}{U_{122}(x, y)}=k(x)$, thus we just have 
to focus on the functions $U$ satisfying

$$
U_{11}(x, y)-k(x) \cdot U_{1}(x, y)=0,
$$

whose general solution is

$$
U(x, y)=F(x) \cdot G(y)+h(y),
$$

where $F$ is such that $F^{\prime \prime} / F^{\prime}=k, G$ and $h$ are arbitrary functions. We only have to require that $G$ keeps a constant sign in order for $U_{1}(x, y)$ to remain positive for all $x$ and $y$. As in the proof of proposition 1 , one can easily check that statement ( $l$ ) actually holds for $U$ given by (16).

Now, to end with this demonstration, let us focus on the case where $U_{122}=0$. A straightforward integration of this equation leads to

$$
U(x, y)=F(x) \cdot y+h(y)+g(x) .
$$

Again, it is only a matter of simple algebraic manipulations to show that $(l)$ is satisfied for such a utility function.

Proof of proposition 3. The bivariate utility function $U(x, y)=F(x) \cdot y+h(y)+g(x)$ can be specified by the knowledge of three conditional utility functions $U\left(x_{0}, y\right), U\left(x, y_{0}\right)$ and $U\left(x, y_{1}\right)$ for arbitrary $x_{0}$, $y_{0}$ and $y_{1} \neq y_{0}$. Indeed, we can define $y_{0}$ and $y_{1}$ such that $U\left(x_{0}, y_{1}\right)>U\left(x_{0}, y_{0}\right)$ and then arbitrarily set the origin and units of measures of $U(x, y)$ by

$$
U\left(x_{0}, y_{0}\right)=0, \quad \text { and } \quad U\left(x_{0}, y_{1}\right)=1 .
$$

Considering the three conditional utility functions

$$
\begin{aligned}
& U\left(x_{0}, y\right)=F\left(x_{0}\right) \cdot y+h(y)+g\left(x_{0}\right), \\
& U\left(x, y_{0}\right)=F(x) \cdot y_{0}+h\left(y_{0}\right)+g(x), \\
& U\left(x, y_{1}\right)=F(x) \cdot y_{1}+h\left(y_{1}\right)+g(x),
\end{aligned}
$$


we can solve for $F(x), h(y)$ and $g(x)$ which yields

$$
\begin{aligned}
F(x) & =\frac{U\left(x, y_{1}\right)-U\left(x, y_{0}\right)}{y_{1}-y_{0}}-\frac{h\left(y_{1}\right)-h\left(y_{0}\right)}{y_{1}-y_{0}} \\
h(y) & =U\left(x_{0}, y\right)-\frac{y-y_{0}}{y_{1}-y_{0}}-\frac{h\left(y_{1}\right)-h\left(y_{0}\right)}{y_{1}-y_{0}} \cdot y+\frac{h\left(y_{0}\right) \cdot y_{0}-h\left(y_{1}\right) \cdot y_{0}}{y_{1}-y_{0}} \\
g(x) & =\frac{U\left(x, y_{0}\right) \cdot y_{1}-U\left(x, y_{1}\right) \cdot y_{0}}{y_{1}-y_{0}}-\frac{h\left(y_{0}\right) \cdot y_{0}-h\left(y_{1}\right) \cdot y_{0}}{y_{1}-y_{0}}
\end{aligned}
$$

By substitution in the expression of $U(x, y)$ given by proposition 2 , we get the result stated in proposition 3.

\section{References}

Bleichrodt, H., D. Crainich, L. Eeckhoudt. 2003. Comorbidities and the willingness to pay for health improvements. Journal of Public Economics 87, 2399-2406.

Busch T., V. H. Hoffmann. 2007. Emerging Carbon Constraints for Corporate Risk Management. Ecological Economics 62, 518-528.

Chhaochharia, V., Y. Grinstein. 2007. Corporate governance and firm value: The impact of the 2002 governance rules. Journal of Finance 62, 1789-1825.

Eeckhoudt, L., C. Gollier, H. Schlesinger. 2005. Economic and Financial Decisions under Risk. Princeton University Press.

Eeckhoudt, L., B. Rey, H. Schlesinger. 2007. A good sign for multivariate risk taking. Management Science 53, 117-124.

Epstein, L. G., S. M. Tanny. 1980. Increasing generalized correlation: A definition and some economic consequences. Canadian Journal of Economics 13, 16-34.

Fishburn, P. C. 1967. Interdependence and additivity in multivariate, unidimensional expected utility theory. International Economic Review 8, 335-342.

Franke, G., H. Schlesinger, R. Stapleton. 2006. Multiplicative Background Risk. Management Science 52, 146-153.

Gollier, C. 2001. The Economics of risk and Time. MIT Press.

Gollier, C., H. Schlesinger. 2003. Preserving preference rankings under background risk. Economics Letters 80, 337-341.

Keeney, R. L. 1971. Utility Independence and Preferences for Multiattributed Consequences. Operations Research 19, 875-893. 
Keeney, R. L. 1973. Risk independence and multiattributted utility functions. Econometrica 41, 27-34.

Keeney, R. L. and H. Raiffa. 1976. Decisions with Multiple Objectives: Preferences and Value Tradeoffs. Wiley.

Kihlstrom, R., D. Romer, S. William. 1981. Risk aversion with random initial wealth. Econometrica 49, 911-920.

Leskinen, P. and J. Kangas. 2005. Rank reversals in multi-criteria decision analysis with statistical modelling of ratio-scale pairwise comparisons. Journal of the Operational Research Society 56, 855861.

Mullin, C., A. Malani. 2004. Assessing the merits of reallocation under joint and several Liability, with an application to asbestos litigation. Working Paper. Chicago Graduate School of Business.

Nachman, D. C. 1982. Preservation of 'more risk averse' under expectations. Journal of Economic Theory $\mathbf{2 8}, 361-368$.

Pollak, R. A. 1967. Additive Von Neuman-Morgenstern utility functions. Econometrica 35, 485-494.

Richard, S. 1975. Multivariate risk aversion, utility independence and separable utility functions. Management Science 22, 12-21. 